\title{
ORIGINAL
}

\section{Cervical root sonography to differentiate multifocal motor neuropathy from ALS}

\author{
Hiroyuki Nodera, Yuishin Izumi, Naoko Takamatsu, and Ryuji Kaji \\ Department of Neurology, Tokushima University, Tokushima, Japan
}

\begin{abstract}
To explore suggestive evidence of focal proximal demyelination in multifocal motor neuropathy (MMN) without overt evidence of conduction block, we conducted sonographical assessment of cervical nerve roots in 9 MMN patients, 22 ALS patients, and 17 control subjects. The mean diameters of the C5 and C6 roots in MMN patients were significantly larger than those in ALS and controls, especially on the clinically dominant side. Although non-specific, sonography can be a potentially useful diagnostic procedure to support the diagnosis of MIMN, even when overt conduction block is lacking. J. Med. Invest. 63 : 104-107, February, 2016
\end{abstract}

Keywords : multifocal motor neuropathy, ALS, Nerve root sonography

\section{INTRODUCTION}

Multifocal motor neuropathy (MMN) is a demyelinating neuropathy characterized by predominant involvement of motor nerves. MMN can mimic ALS and other lower motor neuron diseases, but, as MMN is treatable by intravenous immune globulin (IVIg) and other immunomodulating therapies (1), its differential diagnosis is important. The diagnosis of MMN is particularly difficult when its diagnostic hallmarks, conduction block (CB) and anti-ganglioside antibody are not demonstrable. In a recent study, $\mathrm{CB}$ was identified in only $60 \%$ of MMN patients (2). Sonography of the peripheral nerves was recently reported to detect signs of widespread demyelination including in proximal nerve segments of MMN patients with $\mathrm{CB}$ (3). Thus, the aim of the present study was to assess whether sonography of the nerve roots can identify nerve hypertrophy as a suggestive evidence of demyelination in MMN patients without $\mathrm{CB}$ and further, to help differentiate MMN from ALS. ALS was chosen for comparison because MMN is still one of the most common masqueraders of ALS when upper motor neuron sign is not obvious.

\section{SUBJECTS AND METHODS}

\section{Subjects}

Recordings were made from nine patients with MMN (all men), 22 with ALS (15 men), and from 17 normal controls (11 men) in Tokushima University Hospital, Japan. All the patients with MMN and ALS were ambulatory. The inclusion criteria for the patients with MMN were the following : (A) progressive muscle atrophy and weakness involving only a few peripheral nerves or roots, without sensory involvement, (B) lack of upper motor neuron signs, pathological reflexes, or bulbar symptoms/signs, (C) the lack of radiological abnormality of the cervical spine in an MRI scan, (D) lack of $\mathrm{CB}$ according to published electrodiagnostic criteria (4), and (E) most importantly, the presence of clinical improvement of

Received for publication November 19, 2015 ; accepted December 28, 2015.

Address correspondence and reprint requests to Hiroyuki Nodera, MD, Department of Neurology, Tokushima University, 3-18-15 Kuramotocho, Tokushima-City 770-8503 Japan and Fax : +81-88-633-7208. the affected limb with IVIg therapy ( $2 \mathrm{~g} / \mathrm{kg}$ given over 5 consecutive days), defined as the improvement of the pre- versus post-therapeutic modified MRC score (1-5 scale including 4- and 4+ grades) equal to or greater than 2 in at least two of the affected muscles $(5,6)$. The mean duration of our clinical follow up of the MMN patients was 42.3 months (range : 18-107 months) and all the patients remained ambulatory. The mean number of patients undergoing IVIg treatment was 9.9 (range : 4-17) with reproducible post-therapeutic improvement.

Inclusion criterion for the ALS group was to meet either the clinically definite, or clinically probable (with or without laboratory support) revised El Escorial ALS criteria. Of note, our ALS patients had predominantly lower motor involvement at the time of the sonographic assessment, thus its clinical distinction from MMN was not straightforward. The control group was made up of individuals with no neurological symptoms or signs. Either MRI or plain four-angle $x$-ray of the cervical spine was performed in the control subjects to confirm the lack of significant degenerative spine disease. All the patients and control subjects gave informed consent to participate in the study. This study was performed in accordance with the principles embodied in the Declaration of Helsinki and was approved by the Institutional Review Board at Tokushima University.

\section{Nerve conduction study}

Nerve conduction studies were performed with percutaneous stimulating and recording electrodes (Viking IV, Nicolet, Madison, USA). Compound muscle action potentials (CMAP) were elicited with distal and proximal stimulation of the median nerve (at the wrist with $7 \mathrm{~cm}$ stimulating-recording distance and at the elbow) and of the ulnar nerve (at the wrist with $7 \mathrm{~cm}$ stimulating-recording distance and on the forearm below the elbow). Distal motor latencies (DL) and motor nerve conduction velocities (CV) were then computed. Sixteen to 32 stimulations at $1 \mathrm{~Hz}$ were given to elicit at least $10 \mathrm{~F}$-waves and their minimum latency was recorded. Additional sites of stimulation (axilla and Erb's point for the median nerve and above the elbow ; axilla and Erb's point for the ulnar nerve) demonstrated neither $\mathrm{CB}$ nor temporal dispersion in any of the subjects. Tibial and peroneal nerves were also stimulated according to standard techniques, and DLs and CVs were within normal limits in all subjects. 


\section{Sonography}

The cervical roots were visualized by high-resolution echosonography (Nemio, Toshiba, Japan) using an $11-\mathrm{MHz}$ linear probe. The subjects were examined in the supine position with the head turned upward. By placing the probe on the lateral aspect of the neck, a coronal oblique plane enabled a longitudinal view of the cervical roots, which were seen as hypoechoic structures, and easy measuring of their diameters at a point $2 \mathrm{~cm}$ distal to the transverse process. The $\mathrm{C} 6$ nerve root was easily identified, as the neighboring $\mathrm{C} 6$ vertebra is characterized by a vertebral artery passing through its transverse foramina and by its prominent anterior tubercle compared with that of the $\mathrm{C} 7$ vertebra. The $\mathrm{C} 5$ and $\mathrm{C} 6$ nerve roots were identified and their diameters measured. As the $\mathrm{C} 7$ and $\mathrm{C} 8$ roots may not be visualized in some patients, especially in obese individuals, we analyzed only $\mathrm{C} 5$ and $\mathrm{C} 6$ roots. The nerve roots appeared to be round, thus the measurements were not influenced by flattening. As the lower cervical roots were deep and their measurements possibly inaccurate, they were excluded from the present study. The sonography technician was blind to the clinical diagnoses.
Statistical analysis

ANOVA withBonferroni post hoc analysis (for comparison among MMN, ALS, and control groups) was used for data analysis (SPSS 20.0, Chicago, U.S.A.). $P$-values less than 0.05 were considered to be significant.

\section{RESULTS}

The clinical and electrodiagnostic data are summarized in Table 1. The average age of the MMN patients was slightly less than that of the other two groups due to one young patient. Other than the male dominancy in MMN, the clinical features were similar among the groups. In MMN, two patients had shoulder involvement, five had predominantly forearm and finger involvement, and one had diffuse arm weakness. There were no significant electrodiagnostic differences between the MMN and ALS patients. Among the control subjects, eight had cervical MRI and nine had plain cervical x-ray, none of whom showed evidence of significant degenerative spine disease.

Figure $1 \mathrm{~A}$ shows the sonographic finding of an MMN patient with a thick $\mathrm{C} 6$ nerve root suggestive of demyelination. In Figure 1B,

Table 1. Summary of patient characteristics and electrodiagnostic features of the CMAP.

\begin{tabular}{|l|l|l|l|l|}
\hline Characteristic/Feature & Nerve & MMN & ALS & Control \\
\hline Age (range) & & $49.1(16-61)$ & $60.3(47-77)$ & $62.0(23-79)$ \\
\hline N (male/female) & & $9(9 / 0) *$ & $22(15 / 7)$ & $17(11 / 6)$ \\
\hline \multirow{2}{*}{ Distal latency of CMAP (ms) } & median & $4.0 \pm 0.5$ & $4.2 \pm 0.6$ & \\
\cline { 2 - 5 } & ulnar & $3.2 \pm 0.5$ & $3.6 \pm 0.7$ & \\
\hline \multirow{2}{*}{ Amplitude of CMAP (mV) } & median & $4.2 \pm 2.2$ & $2.4 \pm 1.6$ & \\
\cline { 2 - 5 } & ulnar & $5.6 \pm 2.3$ & $4.2 \pm 3.3$ & \\
\hline \multirow{2}{*}{ Conduction velocity (m/s) } & median & $57.0 \pm 3.9$ & $55.1 \pm 4.7$ & \\
\cline { 2 - 5 } & ulnar & $58.4 \pm 4.4$ & $58.0 \pm 7.3$ & \\
\hline \multirow{2}{*}{ Minimum F-latency (ms) } & median & $28.0 \pm 3.0$ & $29.1 \pm 5.3$ & \\
\cline { 2 - 5 } & ulnar & $27.8 \pm 1.8$ & $28.7 \pm 3.2$ & \\
\hline
\end{tabular}

$* P<0.05$ male/female compared with controls. Electrodiagnostic study was performed on the clinically dominant side.
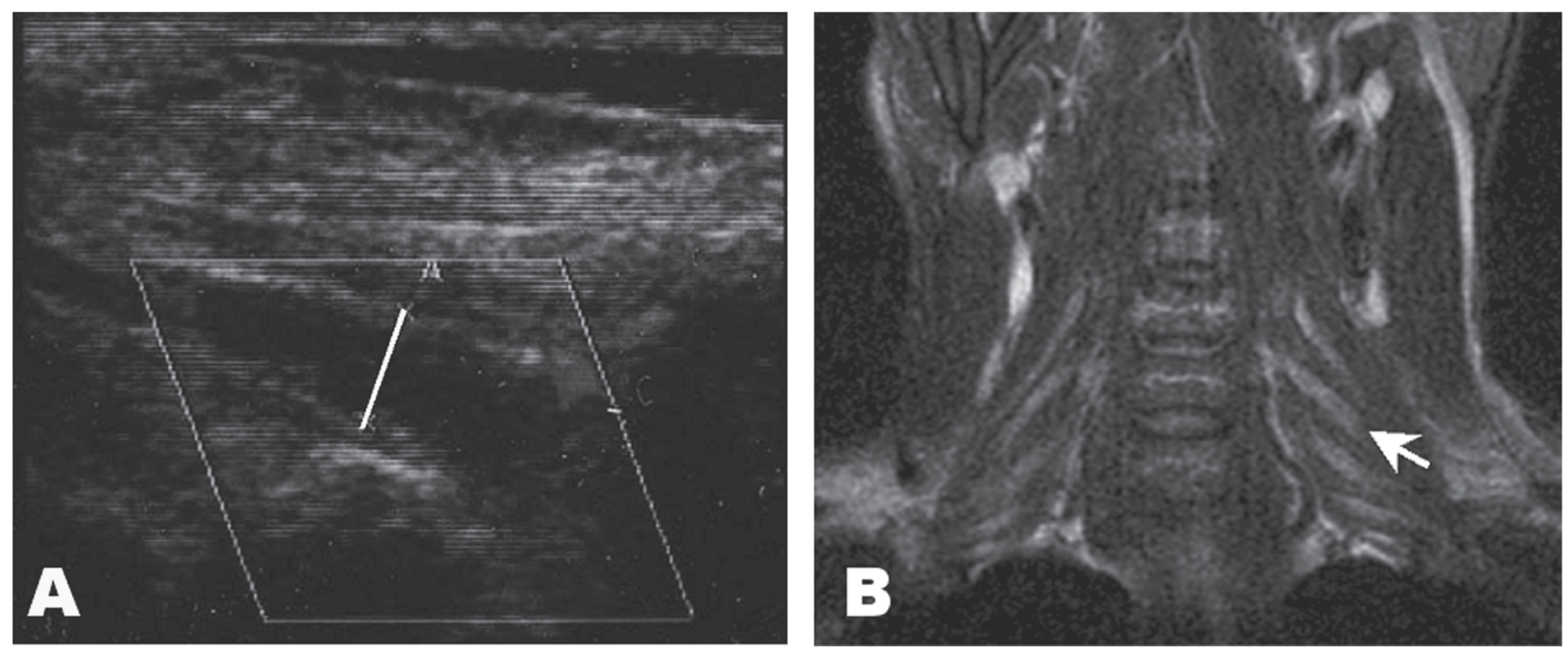

Figure 1. Cervical root swelling in an MMN patient. (A) Sonographic image of the left $\mathrm{C} 6$ nerve root of a patient with multifocal motor neuropathy $(\mathrm{MMN})$. The root diameter, just distal to the dorsal root ganglia, was thicker $(4.9 \mathrm{~mm})$ than the normal upper limit $(4 \mathrm{~mm})$ consistent with demyelination. (B) The T2-weighted MRI image of the same MMN patient demonstrated a thick left C6 nerve root (arrow) with a diameter of $4.7 \mathrm{~mm}$, comparable with that obtained by ultrasound. Note the thicker nerve roots on the symptomatic left side compared with those on the right. 
the MRI imaging corroborated the sonographic finding. As a group, the patients with MMN had larger nerve root diameters than those with ALS or the control groups, with the normal upper limit of both the $\mathrm{C} 5$ and $\mathrm{C} 6$ diameters being $4.0 \mathrm{~mm}$ (Figures 2 and 3). Of note, the root diameters in MMN were asymmetric, with the clinically affected roots tending to show increased diameters. In MNN patients, the percentage of thick $\mathrm{C} 6$ nerve roots was higher than that of C5 roots ( $77 \%$ vs. $55 \%$, respectively) on the clinically dominant side, likely reflecting the clinical involvement in our MMN patients. By contrast, the root diameters of some ALS patients were thinner than those of controls, suggesting axonal degeneration.

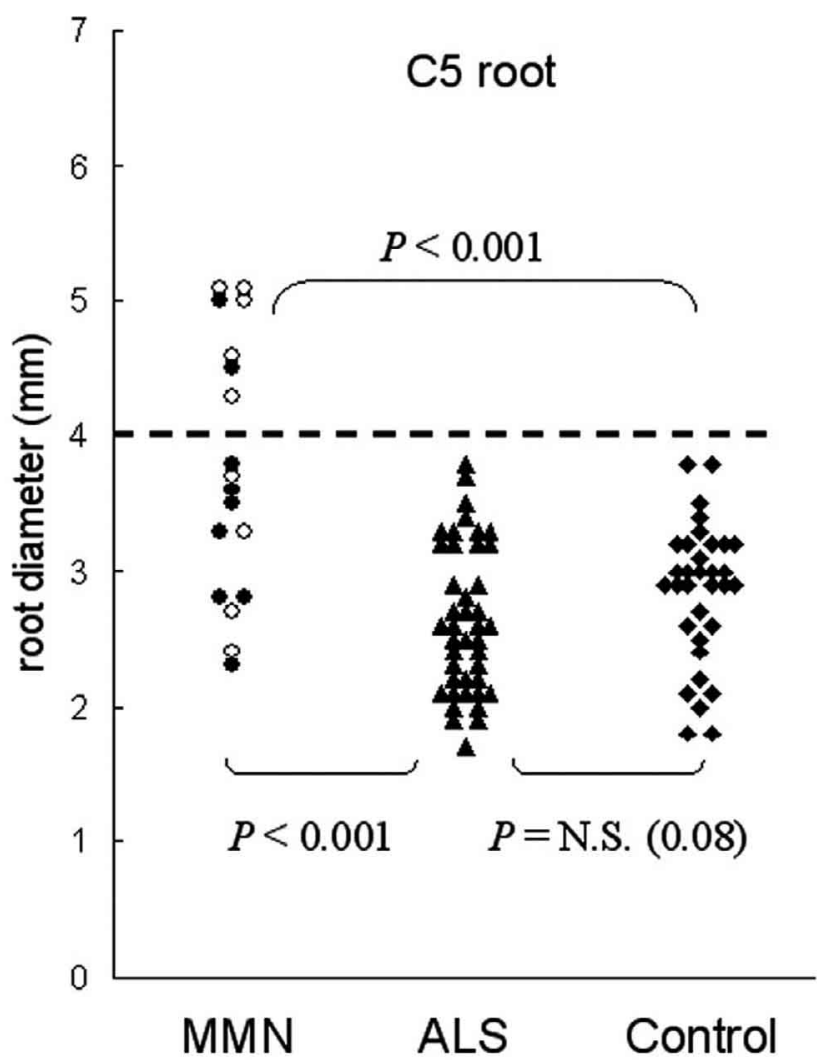

Figure 2. The diameters of the C5 nerve roots in MMN, ALS and control groups. The 95-percentile value in the control group, taken as the normal upper limit, was $4 \mathrm{~mm}$ (dotted lines). In MMN, the mean diameters were larger than in both the control and ALS groups. The increased thickness was more significant on the clinically weaker side (open circles).

\section{DISCUSSION}

In the present study, we have shown that sonography can detect focal thickness of a cervical nerve root in MMN to suggest demyelination that can differentiate MMN from ALS, which has been demonstrated to have pathologically thinner nerve roots due to atrophy (7), and from normal controls.

The role of peripheral nerve sonography to detect nerve enlargement and changes in echogenicity have been previously described. Demyelinating neuropathies such as chronic inflammatory demyelinating polyneuropathy (CIDP) and Charcot-Marie-Tooth disease show diffusely thickened peripheral nerves and nerve roots $(8,9)$. MRI and sonography are also able to detect focal enlargement of nerves, plexuses and nerve roots due to infection, inflammation, infiltration and neoplasm (10). Recent comparison between MRI

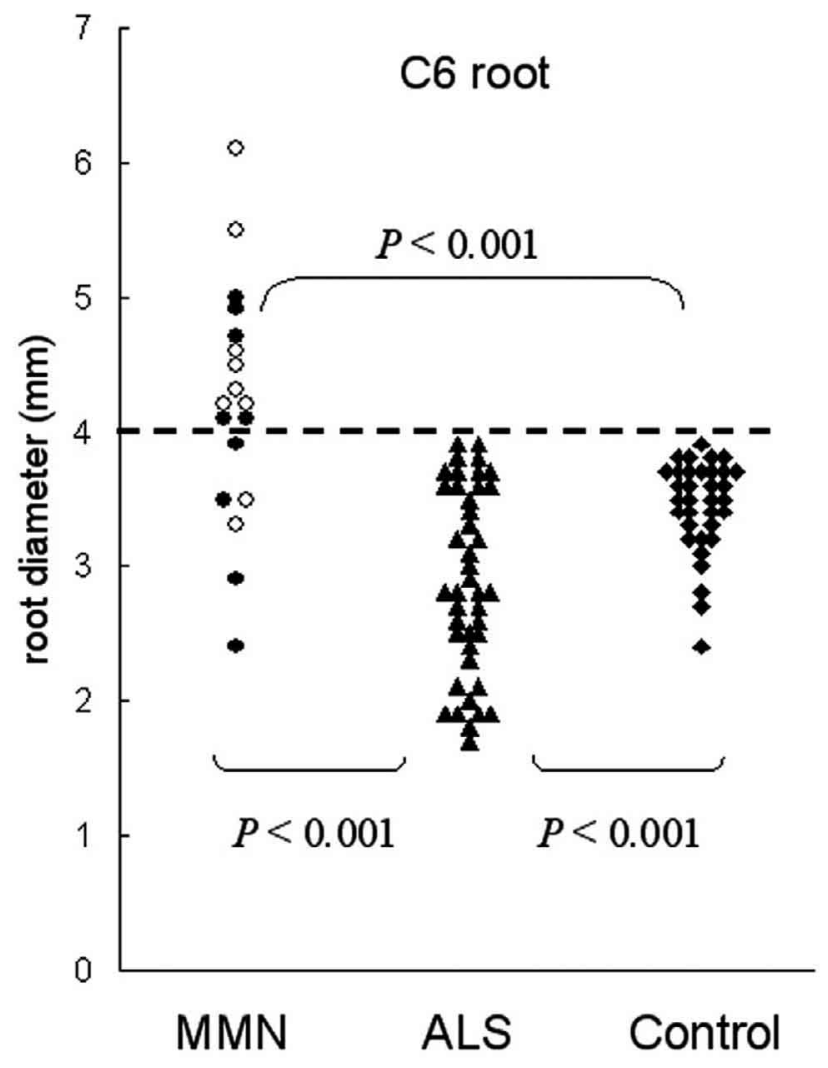

Figure 3. The diameters of the C5 nerve roots in MMN, ALS and control groups. Similar to the C5 root (Figure 2), the mean diameters were larger in MMN than in both the control and ALS groups. The root diameters of some of the ALS patients were thinner than the controls, suggestive of axonal degeneration.

and sonography in CIDP showed that the two techniques are relevant in detecting microstructure such as nerve fascicles, such that sonography could replace MRI in daily practice due to its affordability (11).

In $\mathrm{MMN}$, peripheral nerves at the sites of $\mathrm{CB}$ and at electrodiagnostically normal sites were reported to be demyelinated $(12,13)$ and demonstrated increased diameters by MRI (14). This finding was confirmed by a recent report employing high resolution sonography in MMN patients $(3,15,16)$, but its utility for differential diagnosis, especially in MMN patients without solid diagnostic evidence (e.g., CB and/or anti-ganglioside antibodies), had been little explored. We have thus demonstrated that, in patients with otherwise typical MMN but no overt $\mathrm{CB}$, sonography can provide evidence of demyelination. Sonography would be particularly useful in diagnosing a patient with predominantly lower motor neuron involvement and in predicting the therapeutic efficacy of intravenous immunoglobuiln in MMN (17).

That said, some of the MMN patients in the present study had normal root diameters and several explanations are possible : (A) the demyelination was focal and the examined $\mathrm{C} 5$ or $\mathrm{C} 6$ nerve roots were not significantly affected, (B) the roots were initially thickened, but later became thinner due to secondary axonal degeneration, (C) the focal demyelination existed somewhere other than at the root level, and (D) the presence of chronic motor axonal neuropathy, which shows no clear evidence of demyelination, but has only axonal degeneration (i.e., thinning of the peripheral nerve) (18).

Because sonographic detection of nerve thickness has also been reported in other demyelinating neuropathies, the finding should 
be interpreted according to the clinical information. A novice echosonographer may not be able to obtain the appropriate crosssectional and longitudinal images, leading to either over- or underdiagnosis of nerve swelling and atrophy. However, under an experienced hand, measurements of a nerve size have been reported to have high intra- and inter-observer reliability. By using similar recording methods, Sugimoto, et al. reported coefficients of variation in normal individuals. Their intra- and inter-observer coefficients of variation of the $\mathrm{C} 5$ - $\mathrm{C} 7$ nerve roots ranged from 5.5 to $14.3 \%$ (19). Another limitation is that we did not compare the sizes of the nerve roots by sonography and MRI in all the subjects. Although MRI might have lower operator-dependency than sonography, MRI might not depict nerve roots with the largest cross-sectional plane, thus accurate comparison might not be possible.

Despite this limitation and its non-specificity, sonographic detection of nerve thickness may suggest focal demyelination and thus may support the diagnosis of MMN, especially in those cases without overt CB.(2) Further study is needed to elucidate the locations of the "electrically silent" nerve swelling and thus suggest a diagnostic protocol to detect such likely demyelinating lesions.

\section{ACKNOWLEDGEMENTS}

We thank Drs. Masafumi Harada and Motohiro Hirasawa for obtaining and interpreting the MRI scan of the cervical nerve roots.

\section{REFERENCES}

1. Kaji R, Shibasaki H, Kimura J : Multifocal demyelinating motor neuropathy : cranial nerve involvement and immunoglobulin therapy. Neurology $42:$ 506-9, 1992

2. Delmont E, Azulay JP, Giorgi R, Attarian S, Verschueren A, Uzenot D, Pouget J : Multifocal motor neuropathy with and without conduction block : a single entity? Neurology 67 : 5926,2006

3. Beekman R, van den Berg LH, Franssen H, Visser LH, van Asseldonk JT, Wokke JH : Ultrasonography shows extensive nerve enlargements in multifocal motor neuropathy. Neurology $65: 305-7,2005$

4. AAEM : Consensus criteria for the diagnosis of partial conduction block. Muscle Nerve 22 : S225-S9, 1999

5. Miyashiro A, Matsui N, Shimatani Y, Nodera H, Izumi Y, Kuwabara S, Imai T, Baba M, Komori T, Sonoo M, Mezaki T, Kawamata J, Hitomi T, Kawamata J, Hitomi T, Kohara N, Arimura K, Hashimoto S, Arisawa K, Kusunoki S, Kaji R, Japanese Multifocal Motor Neuropathy Study G : Are multifocal motor neuropathy patients underdiagnosed? An epidemiological survey in Japan. Muscle Nerve 49 : 357-61, 2014

6. Busby M, Donaghy M : Chronic dysimmune neuropathy. A subclassification based upon the clinical features of 102 patients. J Neurol 250 : 714-24, 2003
7. Sobue G, Matsuoka Y, Mukai E, Takayanagi T, Sobue I : Pathology of myelinated fibers in cervical and lumbar ventral spinal roots in amyotrophic lateral sclerosis. J Neurol Sci 50 : 413-21, 1981

8. Di Pasquale A, Morino S, Loreti S, Bucci E, Vanacore N, Antonini G : Peripheral nerve ultrasound changes in CIDP and correlations with nerve conduction velocity. Neurology 84 : 803-9, 2015

9. Noto YI, Shiga K, Tsuji Y, Mizuta I, Higuchi Y, Hashiguchi A, Takashima H, Nakagawa M, Mizuno T: Nerve ultrasound depicts peripheral nerve enlargement in patients with genetically distinct Charcot-Marie-Tooth disease. J Neurol Neurosurg Psychiatry, 2014

10. Khadilkar SV, Yadav RS, Soni G : A practical approach to enlargement of nerves, plexuses and roots. Pract Neurol 15 : 10515,2015

11. Pitarokoili K, Schlamann M, Kerasnoudis A, Gold R, Yoon MS : Comparison of clinical, electrophysiological, sonographic and MRI features in CIDP. J Neurol Sci 357 : 198-203, 2015

12. Kaji R, Oka N, Tsuji T, Mezaki T, Nishio T, Akiguchi I, Kimura $\mathrm{J}$ : Pathological findings at the site of conduction block in multifocal motor neuropathy. Ann Neurol 33 : 152-8, 1993

13. Taylor BV, Dyck PJ, Engelstad J, Gruener G, Grant I : Multifocal motor neuropathy : pathologic alterations at the site of conduction block. J Neuropathol Exp Neurol 63 : 129-37, 2004

14. Van Es HW, Van den Berg LH, Franssen H, Witkamp TD, Ramos LM, Notermans NC, Feldberg MA, Wokke JH : Magnetic resonance imaging of the brachial plexus in patients with multifocal motor neuropathy. Neurology 48 : 1218-24, 1997

15. Grimm A, Decard BF, Athanasopoulou I, Schweikert K, Sinnreich M, Axer H : Nerve ultrasound for differentiation between amyotrophic lateral sclerosis and multifocal motor neuropathy. J Neurol 262 : 870-80, 2015

16. Loewenbruck KF, Liesenberg J, Dittrich M, Schafer J, Patzner B, Trausch B, Machetanz J, Hermann A, Storch A : Nerve ultrasound in the differentiation of multifocal motor neuropathy $(\mathrm{MMN})$ and amyotrophic lateral sclerosis with predominant lower motor neuron disease (ALS/LMND). J Neurol, 2015

17. Nobile-Orazio E, Cappellari A, Meucci N, Carpo M, Terenghi F, Bersano A, Priori A, Barbieri S, Scarlato G : Multifocal motor neuropathy : clinical and immunological features and response to IVIg in relation to the presence and degree of motor conduction block. J Neurol Neurosurg Psychiatry 72 : 761-6, 2002

18. Kaji R, Kusunoki S, Mizutani K, Oka N, Kojima Y, Kohara N, Kimura J : Chronic motor axonal neuropathy associated with antibodies monospecific for $\mathrm{N}$-acetylgalactosaminyl GD1a. Muscle Nerve 23 : 702-6, 2000

19. Sugimoto T, Ochi K, Hosomi N, Mukai T, Ueno H, Takahashi T, Ohtsuki T, Kohriyama T, Matsumoto M : Ultrasonographic reference sizes of the median and ulnar nerves and the cervical nerve roots in healthy Japanese adults. Ultrasound in medicine \& biology 39 : 1560-70, 2013 